\title{
INFLUÊNCIA DE DANOS FOLIARES DE LAGARTA-DO-CARTUCHO EM HÍBRIDOS DE MILHO TRANSGÊNICOS E CONVENCIONAIS
}

\author{
THE INFLUENCE OF FOLIAR DAMAGE BY THE FALL ARMYWORM ON \\ TRANSGENIC AND CONVENTIONAL MAIZE HYBRIDS
}

\begin{abstract}
Cristiani Santos Bernini ${ }^{1 *}$, Thais Alves Oliveira ${ }^{2}$, Zulema Netto Figueiredo ${ }^{3}$
${ }^{1}$ Professora doutora do curso de Agronomia da Universidade do Estado do Mato Grosso - UNEMAT (Cáceres/MT).

2 Discente do curso de Agronomia da Universidade do Estado de Mato Grosso UNEMAT (Cáceres/MT).

${ }^{3}$ Professora doutora adjunta do curso de Agronomia da Universidade do Estado do Mato Grosso - UNEMAT (Cáceres/MT).

* Avenida Santos Dumont, s/n, Cidade Universitária, Departamento de Agronomia, Cáceres/MT, CEP: 78.200-000 E-mail: cristiani.bernini@unemat.br
\end{abstract}

Submetido em: 05/09/2019; Aceito em:10/12/2019.

\section{RESUMO}

Com o grande aparecimento de doenças e pragas na cultura do milho, a melhor solução para preservar produtividade e qualidade do milho tem sido o uso de tecnologia Bt (Bacillus thuringiensis), sendo uma tática que diminui os inconvenientes advindos de inseticidas. O presente trabalho teve por objetivo avaliar a incidência de Spodoptera frugiperda em híbridos de milho transgênicos e convencionais e sua resistência fenotípica. $O$ experimento foi realizado em Cáceres - MT, na $2^{\mathrm{a}}$ safra de 2018, em delineamento experimental de blocos ao acaso, com três repetições, avaliando-se seis híbridos na versão convencional e treze transgênicos. Para a avaliação dos danos foliares ocasionados por Spodoptera frugiperda, utilizou-se de uma escala visual de notas, com variação de 0 a 9 . Os maiores danos ocasionados por $S$. frugiperda foram nos híbridos convencionais AL BANDEIRANTE, $F_{2}$ DAS2B710 x $F_{2}$ A2555, $F_{2}$ DAS2B710 $x$ $\mathrm{F}_{2} 30 \mathrm{~F} 35$ e $\mathrm{F}_{2} \mathrm{FORT} \times \mathrm{F}_{2} \mathrm{AG} 8060$ e de menor dano no híbrido convencional $\mathrm{F}_{2} \mathrm{~A} 2555 \times \mathrm{F}_{2} \mathrm{FORT}$. Híbridos transgênicos com maiores danos por $S$. frugiperda foram DKB 230 PRO3, MG580 e DKB 290 PRO 3 e de menor dano foliar o híbrido transgênico AG 8088 VT PRO com tecnologia Viptera ${ }^{\circledR}$. Pode-se discriminar híbridos transgênicos resistentes à lagarta-do-cartucho através do monitoramento de resistência à inseto realizado aos DSF 45 e DSF 60.

Palavras-chave: Correlação fenotípica. Milho transgênico. Monitoramento de insetos.

\section{ABSTRACT}

With the large-scale emergence of diseases and pests in maize crops, the best solution to maintain maize yield and quality has been the use of $\mathrm{Bt}$ (Bacillus thuringiensis) technology, since it is a tactic which reduces the issues arising from insecticides. The aim of this study was to evaluate the incidence of Spodoptera 
frugiperda in transgenic and conventional maize hybrids and its phenotypic resistance. The experiment was carried out in Caceres - MT, on the 2nd crop of 2018, in a randomized block design, with three replications, evaluating six hybrids of a conventional version and thirteen transgenics. For the assessment of leaf damage caused by Spodoptera frugiperda, a visual scale of grades was used, with a variation of 0 to 9 . The greatest damage caused by $S$. frugiperda was in the conventional hybrid AL Bandeirante, F2DAS2B710 x F2A2555, F2DAS2B710 $x$ F230F35 and F2FORT $x$ F2AG8060 and the least damage was to the conventional hybrid F2A2555 x F2FORT. Transgenic hybrids with greater S. frugiperda damage were DKB 230 PRO3, MG580 and DKB 290 PRO 3 and the least leaf damage was to the transgenic hybrid AG 8088 VT PRO with Viptera Technology ${ }^{\circledR}$. Transgenic hybrids resistant to fall armyworm can be discriminated by scouting for insect resistance at DSF 45 (45 days) and DSF 60 (60 days).

Keywords: Phenotypic correlation. Transgenic Maize. Scouting for Insects.

\section{INTRODUÇÃO}

A soja e o milho são as principais culturas produzidas no país que apresentam ganho em produção na ordem de 18,4\% e 39,5\%, respectivamente. A Companhia Nacional de Abastecimento (CONAB, 2019) demonstra que, dos 229,75 milhões de toneladas de grãos da safra 2017/2018, aproximadamente 85 milhões de toneladas são de milho (37\%). Em comparação à safra anterior são 12 milhões de toneladas a menos em 97,9 milhões de toneladas, principalmente, pela queda de produção em razão da restrição hídrica no milho segunda safra cultivado nos estados do Paraná e Mato Grosso do Sul, e também a redução de área cultivada com milho primeira safra face as condições econômicas mais favoráveis ao cultivo da soja nesta época do ano, principalmente na região Centro-Sul (CONAB, 2019).

Diante desse cenário, o clima foi fator prejudicial na expressão do potencial máximo da produtividade de híbridos de milho. Com isso, é imprescindível estudar os ambientes de produção como fator de impacto nos híbridos, e sendo favorável, o alto potencial de híbridos é assim dependente do suprimento adequado de água, controle de doenças, insetos e plantas daninhas, além de adequada fertilidade do solo (PEREIRA FILHO; BORGHI, 2018).

A lagarta-do-cartucho Spodoptera frugiperda é a principal praga da cultura de milho no Brasil pertencente à ordem Lepidoptera, família Noctuidae. No milho, o ataque dessa praga ocorre tanto nos cultivos de verão quanto nos de segunda safra. $O$ inseto faz o seu ataque a planta desde a sua emergência, cortando-a rente ao solo e destruindo as espigas em formação, também raspam as folhas e se alojam no cartucho e o período crítico é próximo ao florescimento (CRUZ, 1995).

Uma das principais estratégias de manejo de resistência à insetos a culturas Bt (Bacillus thuringiensis) envolve a combinação de dois conceitos independentes para retardar a evolução de resistência: alta expressão de genes cry (alta dose) e o uso de áreas de refúgio. A alta expressão da proteína inseticida torna ineficaz qualquer mecanismo que confere ao inseto níveis de resistência baixos ou moderados e assume-se que nessa condição a maioria dos 
heterozigotos serão mortos. O refúgio compreende a área onde determinada parcela da população não é exposta à pressão de seleção, ou seja, pode sobreviver, reproduzir e acasalar-se com indivíduos sobreviventes da área Bt e produzir descendentes (heterozigotos - RS) suscetíveis (BERNARDI et al., 2011).

As problemáticas advindas do uso do manejo inadequado das cultivares $\mathrm{Bt}$ foram relatadas inicialmente pelos produtores do Mato Grosso representados pela Associação dos Produtores do Estado do Mato Grosso (APROSOJA$\mathrm{MT} / 2018$ ) que não conseguiam controlar as pragas alvos com apenas a tecnologia, dispendendo de várias aplicações de inseticidas. De acordo com Mendes e Waquil (2009) é possível que o produtor encontre, em condições de campo, as respostas diferenciadas em relação ao controle da lagarta-docartucho com a utilização de eventos diferentes.

Contudo, poucas são as informações a respeito das tecnologias disponibilizadas pelos produtores a cada safra. Pereira Filho e Borghi (2018), realizaram o levantamento das cultivares disponíveis para os produtores, onde $68 \%$ dos materiais apresentaram alguma tecnologia transgênica, enquanto os convencionais representaram $32 \%$ da relação total. Estes dados representam a maior oferta de materiais transgênicos no mercado, o que vem a aumentar ainda mais a necessidade de pesquisas nesta área.

De acordo com Crubelati-Mulati et al. (2019) a adoção de genótipos resistentes representa um método eficiente no controle de pragas, por causar efeitos adversos na biologia dos insetos (antibiose) ou fazendo com que os insetos prefiram uma planta inferior a outro para alimentação (não preferência), devido a características químicas e físicas da planta.

O presente trabalho enfoca a questão sobre o milho híbrido destacando a sua resistência perante o ataque da lagarta-do-cartucho. Com o decorrente aparecimento de doenças e pragas, foram criadas tecnologias de aperfeiçoamento através da biotecnologia e melhoramento vegetal de plantas para se obter novas cultivares de milhos com maior produtividade, qualidade e resistência. Dada a importância econômica que o milho tem para o Brasil e a nível Internacional, é primordial que sejam estudadas formas em que possam aumentar suas características de desenvolvimento agronômico.

Considerando os prejuízos econômicos ocasionados pela lagarta-docartucho na cultura do milho. Objetivou-se avaliar a infestação de Spodoptera frugiperda (J. E. Smith) em híbridos de milho convencionais e transgênicos e seus efeitos em características agronômicas em campo.

\section{MATERIAL E MÉTODOS}

O experimento foi conduzido na Fazenda Ressaca do Grupo Nelore Grendene localizada no município de Cáceres - MT, com as coordenadas geográficas latitude $16^{\circ} 13^{\prime} S$ e longitude $57^{\circ} 70^{\prime} \mathrm{W}$ e altitude $139 \mathrm{~m}$, na $2^{\mathrm{a}}$ safra de 2018.

No experimento foram utilizadas no total 19 cultivares de milho híbridos, onde 13 cultivares são híbridos transgênicos e 6 cultivares são híbridos convencionais (Tabela 1). 
O experimento foi conduzido sob o delineamento experimental de blocos ao acaso com 3 repetições, sendo cada parcela constituídas por duas linhas de $6 \mathrm{~m}$ espaçadas de $1,0 \mathrm{~m}$ entre parcelas e $0,20 \mathrm{~m}$ entre plantas. Os tratos culturais foram de acordo com a necessidade da cultura, não sendo utilizado nenhum tipo de inseticida para controle de lagarta-do-cartucho ou de outros insetos na área.

Para verificar a intensidade dos danos causados pela Spodoptera frugiperda (lagarta-do-cartucho) nas folhas, em condições de infestação natural, a primeira avaliação ocorreu aos 30 dias após a semeadura, quando as plantas encontram-se entre os estádios V6 e V8 (6 a 8 folhas totalmente expandidas). As demais avaliações foram realizadas quinzenalmente após a primeira avaliação até o início do pendoamento das plantas, totalizando três avaliações DSF 30, DSF 45 e DSF 60 (Danos de Spodoptera Foliar).

Tabela 1 - Descrição dos híbridos comerciais de milho.

\begin{tabular}{|c|c|c|c|c|}
\hline Cultivar & $\begin{array}{c}\text { Base } \\
\text { Genética* }\end{array}$ & Tecnologia & Proteína & Empresa \\
\hline MG 580 PW & HS & $\mathrm{Bt}$ & $\begin{array}{c}\text { Cry1F + Cry } 1 \mathrm{~A} .105+ \\
\text { Cry2Ab }\end{array}$ & Morgan \\
\hline MG 600 PW & HS & $\mathrm{Bt}$ & $\begin{array}{c}\text { Cry1F }+ \text { Cry } 1 \mathrm{~A} .105+ \\
\text { Cry } 2 \mathrm{Ab}\end{array}$ & Morgan \\
\hline 30F53 VYH & $\mathrm{HS}$ & $\mathrm{Bt}$ & $\begin{array}{c}\text { Cry1Ab + Cry } 1 F+ \\
\text { Vip3Aa20 }\end{array}$ & Pioneer \\
\hline 30F35 VYH & $\mathrm{HT}$ & $\mathrm{Bt}$ & $\begin{array}{c}\text { Cry1Ab + Cry1F + } \\
\text { Vip3Aa20 }\end{array}$ & Pioneer \\
\hline AG 8088 PRO2 VT & HS & $\mathrm{Bt}$ & Cry1A.105 + Cry2Ab & Agroceres \\
\hline AG 7000 PRO2 & HS & $\mathrm{Bt}$ & Cry $1 A .105+$ Cry2Ab & Agroceres \\
\hline Formula VIP & HS & $\mathrm{Bt}$ & VIP3Aa20 & Syngenta \\
\hline RB 9110 PRO2 & HS & $\mathrm{Bt}$ & Cry1A.105 + Cry2Ab & $\begin{array}{c}\text { Riber } \\
\text { Sementes }\end{array}$ \\
\hline P1680 YH & HS & $\mathrm{Bt}$ & Cy $1 F+$ Cry $1 A B$ & Pioneer \\
\hline DKB 290 PRO 3 & HS & $\mathrm{Bt}$ & $\begin{array}{c}\text { Cry } 1 A .105+\text { Cry2Ab } \\
+ \text { Cry3Bb }\end{array}$ & Dekalb \\
\hline DKB 390 PRO 2 & HS & $\mathrm{Bt}$ & Cry1A.105 + Cry2Ab & Dekalb \\
\hline DKB 230 PRO 3 & HS & $\mathrm{Bt}$ & $\begin{array}{c}\text { Cry } 1 A .105+\text { Cry2Ab } \\
+ \text { Cry3Bb }\end{array}$ & Dekalb \\
\hline DKB 177 PRO 3 & HS & $\mathrm{Bt}$ & $\begin{array}{c}\text { Cry } 1 A .105+\text { Cry2Ab } \\
+ \text { Cry3Bb }\end{array}$ & Dekalb \\
\hline Al Bandeirante & V & Convencional & - & CATI \\
\hline 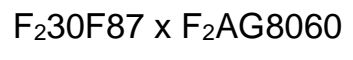 & $\mathrm{HI}$ & Convencional & - & IAC \\
\hline $\mathrm{F}_{2} \mathrm{FORT} \times \mathrm{F}_{2} \mathrm{AG} 8060$ & $\mathrm{HI}$ & Convencional & - & IAC \\
\hline $\begin{array}{c}\mathrm{F}_{2} \mathrm{DAS} 2 \mathrm{~B} 710 \mathrm{x} \\
\mathrm{F}_{2} \mathrm{~A} 255\end{array}$ & $\mathrm{HI}$ & Convencional & - & IAC \\
\hline $\begin{array}{c}\mathrm{F}_{2} \mathrm{DAS} 2 \mathrm{~B} 710 \mathrm{x} \\
\mathrm{F}_{2} 30 \mathrm{~F} 35\end{array}$ & $\mathrm{HI}$ & Convencional & - & IAC \\
\hline $\mathrm{F}_{2} \mathrm{~A} 2555 \times \mathrm{F}_{2} \mathrm{FORT}$ & $\mathrm{HI}$ & Convencional & - & IAC \\
\hline
\end{tabular}

Notas: *HS: híbrido simples; HT: híbrido triplo; HI: híbrido intervarietal; V: variedade Fonte: Oliveira, 2018. 
As avaliações de danos causados pela Spodoptera frugiperda foram feitas por três avaliadores, cada um amostrando 10 plantas ao acaso por parcela e, por meio de uma escala de notas visuais (Tabela 2), atribuíram-se notas que variaram de 0 (sem danos) a 9 (cartucho totalmente destruído), de acordo com Fernandes et al. (2003).

Tabela 2 - Escala de notas de danos causados por Spodoptera frugiperda nas folhas em híbridos de milho (de acordo com FERNANDES et al. (2003), adaptada de DAVIS et al., (1992)).

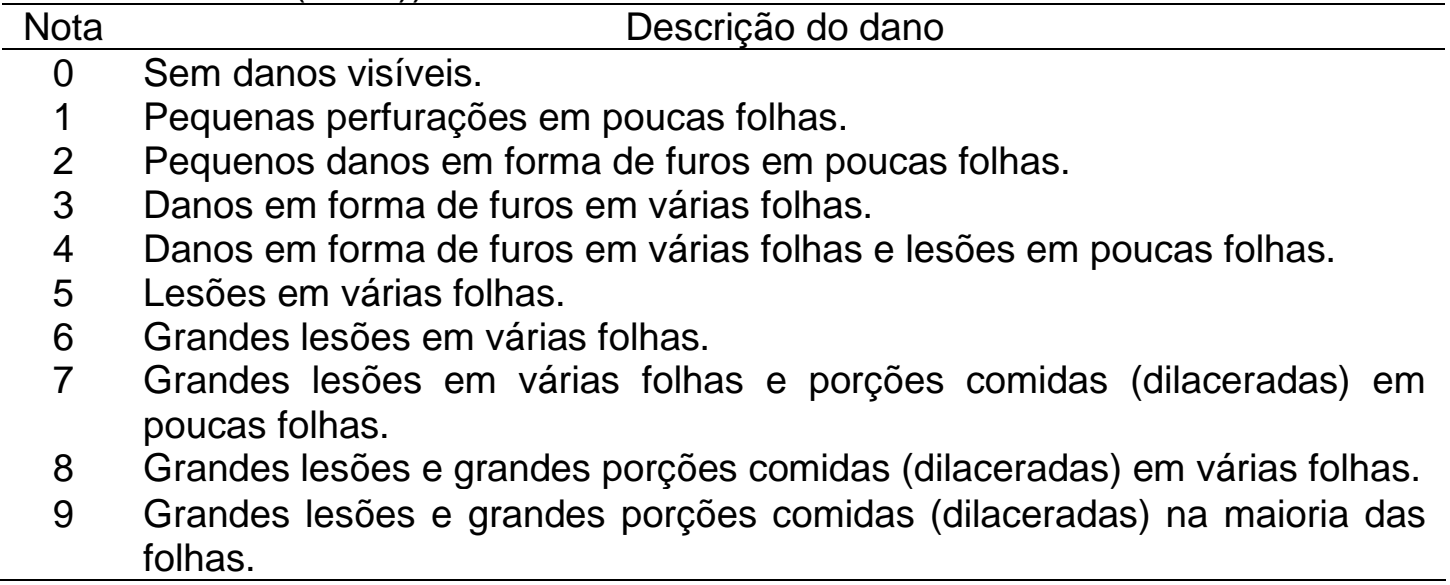

Fonte: Fernandes et al. (2003).

Para as análises dos dados, foram feitas as médias das dez plantas por parcela e posteriormente as médias dos avaliadores. Os dados foram submetidos à análise de variância individuais e conjuntas, considerando o modelo fixo, e os valores médios foram comparados pelo teste de Scott-Knott, ao nível de $5 \%$ de probabilidade de erro. A análise conjunta contém as informações do efeito de tratamentos em híbridos e do contraste tratamentos versus épocas de avaliação. Para atender as necessidades de normalidade e homocedasticidade, antes de proceder as análises de variância, os dados referentes às notas de sintomas, foram submetidos à transformação de $(x+$ $0,5)^{1 / 2}$. A correlação de Pearson foi estimada entre os estádios de avaliação de danos de Spodoptera nas folhas. Todas as análises foram efetuadas empregando o programa estatístico Genes.

\section{RESULTADOS E DISCUSSÃO}

Foram detectadas diferenças significativas entre os tratamentos nas avaliações DSF45 e DSF60. Desse modo, observou-se que a lagarta-docartucho afeta de maneira distinta os híbridos de milho avaliados e que o nível de infestação da lagarta em campo também é variável, condição essa dependente de fatores abióticos e da genética do cultivar.

Os coeficientes de variação (CV) das análises individuais (Tabela 3) foram, em geral, de magnitude baixa a mediana, segundo a classificação proposta por Scapim et al. (1995). Esse resultado evidencia boa precisão na tomada dos dados dos experimentos, o que é bastante desejável, porque permite a obtenção de uma melhor resposta experimental. 
Foram detectadas diferenças significativas entre as épocas de avaliações dos danos ocasionados pela lagarta $(p<0,01)$ (Tabela 4). Verificaram-se diferenças significativas entre os tratamentos indicando variabilidade genética dos híbridos avaliados. $O$ efeito de tratamento versus época de avaliação não foi significativo para 0 ataque de lagarta.

As médias gerais de ataques de lagarta-do-cartucho se encontram na Tabela 5 bem como as correspondentes épocas de avaliações. Os danos provocados foram mais severos nos híbridos convencionais do que nos híbridos transgênicos. Tais valores foram representados por plantas com danos em forma de furos em várias folhas e lesões em poucas folhas nota 3,88 para os híbridos Bt e plantas com lesões em várias folhas nota 4,64 para os híbridos convencionais.

Tabela 3 - Análise de variância individual das notas de ataque de lagarta-docartucho, avaliada em 3 estádios de desenvolvimento do milho correspondendo a 30, 45 e 60 dias após a semeadura, respectivamente, DSF 30, DSF 45, DSF 60. $2^{\mathrm{a}}$ safra de 2018. Cáceres - MT.

\begin{tabular}{lcccc}
\hline \multirow{2}{*}{ FV } & GL & \multicolumn{3}{c}{ Quadrados Médios } \\
\cline { 3 - 5 } & & $\begin{array}{c}\text { (1)DSF 30 } \\
\text { (notas) }\end{array}$ & $\begin{array}{c}\text { (2)DSF 45 } \\
\text { (notas) }\end{array}$ & $\begin{array}{c}(3) \text { DSF 60 } \\
\text { (notas) }\end{array}$ \\
\hline Blocos & 2 & 1,094 & 0,087 & 0,15 \\
Híbridos & 19 & $4,251^{\text {ns }}$ & $10,381^{*}$ & $10,897^{*}$ \\
Resíduo & 38 & 2,896 & 7,197 & 7,404 \\
\hline Média & & 1,69 & 5,1 & 5,47 \\
CV & & 34,22 & 28,22 & 27,11 \\
\hline
\end{tabular}

Notas: ${ }^{\text {ns, **: }}$ não significativo, significativo a $1 \%$, pelo teste $F_{;}^{;(1,2,3)}$ : Dados transformados em $(x+0,5) 1 / 2$.

Fonte: os autores.

Tabela 4 - Análise de variância conjunta avaliada em 3 estádios de desenvolvimento do milho, correspondendo a 30, 45 e 60 dias após a semeadura, respectivamente, DSF30, DSF45 e DSF 60 de ataque de lagarta do cartucho (Spodoptera frugiperda). $2^{\circ}$ safra de 2018. Cáceres - MT.

\begin{tabular}{|c|c|c|}
\hline \multirow[b]{2}{*}{ FV } & \multicolumn{2}{|r|}{ Quadrados Médios } \\
\hline & GL & $\begin{array}{c}\text { Ataque de lagarta } \\
\text { (notas) }\end{array}$ \\
\hline Blocos/Época avaliação & 6 & 0,114 \\
\hline Híbridos & 19 & $3,02^{* *}$ \\
\hline Avaliação & 2 & $131,177^{\star \star}$ \\
\hline Tratamentos x Época avaliação & 38 & $0,600^{\text {ns }}$ \\
\hline Erro & 114 & 0,388 \\
\hline Média & & 4,09 \\
\hline CV & & 29,41 \\
\hline
\end{tabular}

Fonte: os autores. 
A interação tratamentos $x$ época de avaliação não mostrou significância para o caráter estudado (ataque de lagartas), o que possibilita discutir os resultados na média de estádios de desenvolvimento de milho (Tabela 4).

As maiores medias de notas de danos foliares foram verificadas para os híbridos convencionais AL BANDEIRANTE, F2DAS2B710 x F2A2555, $F_{2}$ DAS2B710 x $F_{2} 30 F 35$ e $F_{2} F O R T \times F_{2} A G 8060$ e também os transgênicos DKB 230 PRO3, MG580 PW e DKB 290 PRO 3 que não diferiram estatisticamente dos convencionais pelo teste de média Scott- Knott.

Para Andow (2008) e Bernardi et al. (2011), se o tempo que uma praga levaria para evoluir a resistência à proteína $A$ for de 20 gerações e para a proteína $\mathrm{B}$ de 30 gerações, essa praga pode levar 600 gerações para evoluir a resistência para ambas as proteínas. Assim, para uma planta Bt ser considerada "piramidada", as combinações de proteínas inseticidas expressas devem ser tóxicas para a mesma praga alvo, ou ao contrário, "não-piramidada" indicando que as proteínas inseticidas atuam independentemente, não agindo sobre a mesma praga-alvo. Esse tipo de estratégia, que usa da mistura de agentes de mortalidade, preconiza a utilização de dois ou mais agentes de controle em mistura e exige que os produtos apresentem modos de ação distintos e não possuem resistência cruzada.

Neste trabalho, por exemplo, as proteínas Cry1A.105 + Cry2Ab expressas pelo AG8088 PRO2 VT, conferem resistência a determinados lepidópteros-praga da parte aérea e possuem modo de ação através da ligação em receptores específicos do inseto alvo. Bem como, nas tecnologias VT PRO $3^{\circledR}$ e POWERCORE ${ }^{\mathrm{TM}}$ que apresentam as proteínas Cry $1 \mathrm{~F}$ e Cry1A respectivamente juntas, dessa forma a combinação das proteínas Cry1Ac/Cry1F é uma variedade "não-piramidada" para S. frugiperda (ARAGÃO et al., 2011). Pois a proteína Cry1F é tóxica para a $S$. frugiperda, mas a proteína Cry1Ac não é tóxica para a mesma espécie. Em razão da sobreposição de cultivos que expressam semelhantes proteínas inseticidas, a $S$. frugiperda é o inseto que apresenta maior potencial de evoluir para a resistência tanto às proteínas Cry $1 \mathrm{Ab}$, Cry1F e Cry2Ab2 expressas no em milho Bt quanto as proteínas Cry1Ac, Cry2Ab2 e Cry1F expressas em algodão Bt (BERNARDI et al., 2011).

A menor nota de dano entre os híbridos transgênicos AG 8088 VT PRO que contêm a tecnologia Viptera ${ }^{\circledR}$, porém essa tecnologia não diferiu das outras tecnologias (Tabela 5). Entre os híbridos convencionais, destacando-se 0 $\mathrm{F}_{2} 30 \mathrm{~F} 87$ x $\mathrm{F}_{2} \mathrm{AG} 8060$, $\mathrm{F}_{2} \mathrm{DAS} 2 \mathrm{~B} 710$ x $\mathrm{F}_{2} \mathrm{AG} 8060$ e $\mathrm{F}_{2} \mathrm{~A} 2555$ x $\mathrm{F}_{2} \mathrm{FORT}$ que não diferiram entre si estatisticamente, contudo o híbrido $F_{2} A 2555 \times F_{2} F O R T$ se destaca positivamente apresentando a menor nota 2,55 de danos sofrido pela Spodoptera frugiperda. Esse fato pode ser um indicativo de algum tipo de resistência do tipo não-preferência para alimentação desses híbridos em relação à lagarta-do-cartucho. Sabendo-se que dentro do mesmo grupo de insetos, a atividade de cada toxina é diferenciada.

A resistência do tipo não-preferência ocorre quando a planta é menos utilizada pelo inseto, para alimentação, oviposição, ou abrigo, do que outra planta em igualdade de condições, podendo ser de natureza física, química e/ou morfológica (BOIÇA JUNIOR et al., 2013; MORAES, 2015).

De acordo com os autores Ota et al. (2011) e Moraes (2015), menor dano foliar indica menor consumo pela lagarta-do-cartucho, evidenciando, desse 
modo, resistência do tipo não-preferência para alimentação, e assim, o uso de cultivares com esse tipo de resistência, poderia auxiliar na manutenção da população da praga em níveis moderados, otimizando seu manejo. $O$ monitoramento para verificação de alterações na suscetibilidade dos insetoalvos de proteínas Bt é parte integrante dos programas de MRI (Monitoramento da Resistência de Insetos).

A toxina Bt é expressa continuamente nos tecidos da planta, o que explica a eficácia de controle dessa tecnologia durante todo o ciclo das plantas (SOBERÓN et al., 2009; MORAES, 2015).

Com relação aos dados climáticos dos meses de fevereiro a junho de 2018 foram: precipitação pluvial total e temperaturas máxima e mínima. A temperatura média máxima foi de $29^{\circ} \mathrm{C}$ e a mínima de $18^{\circ} \mathrm{C}$. Para precipitação pluvial total foi obtido valor de $509 \mathrm{~mm}$. Ainda com relação à precipitação, a frequência de ocorrência mostra que os meses de fevereiro e março foram os mais chuvosos e que o período de maior estiagem ocorreu de abril a junho, com apenas $21,6 \%$ do total do pluviométrico do período do experimento. Evidenciando de sobremaneira que o período de déficit hídrico nos estádios iniciais da cultura possibilitou o favorecimento da infestação natural da lagarta-do-cartucho e o maior dano foliar nos híbridos.

$\mathrm{Na}$ Tabela 6 são apresentadas as estimativas de correlação fenotípica entre os caracteres avaliados no presente estudo. Os maiores valores de correlação fenotípica foram positivos entre DSF45 e DSF $60\left(0,95^{\star *}\right)$. O conhecimento da associação entre os caracteres é de grande importância, principalmente, se houver dificuldades na seleção de um desses caracteres devido à baixa herdabilidade e/ou problemas na identificação e mediação. Neste caso, a seleção indireta pode levar progressos mais rápidos que a seleção direta para o caráter desejado (CÂMARA et al., 2007). Diante dos resultados obtidos a seleção para DSF 45 indiretamente seleciona-se a DSF 60, que é bastante interessante no monitoramento de resistência a insetos pois permite diminuir o tempo de precaver o dano aos 45 dias após a semeadura (DAS) na lavoura para assim efetuar um controle químico.

Na pesquisa de Hanashiro (2014), em relação aos danos de pragas com a $2^{2}$ avaliação dos danos de Spodoptera nas folhas (DSF 45), indica que 0 aumento da incidência de ataque de Spodoptera nas folhas durante 0 desenvolvimento da planta pode resultar na redução da produtividade de grãos e que quanto maior o dano que a $S$. frugiperda tenha causado nas folhas de milho menor será o diâmetro da espiga. 
Tabela 5 - Média das avaliações de ataque de lagarta-do-cartucho (Spodoptera frugiperda) avaliada em 3 estádios de desenvolvimento do milho correspondendo a 30, 45 e 60 dias após a semeadura, respectivamente, DSF30, DSF45 e DSF 60. $2^{\circ}$ safra de 2018. Cáceres - MT.

\begin{tabular}{|c|c|c|c|c|}
\hline Híbridos de Milho & $\begin{array}{l}\text { (1)DSF } 30 \\
\text { (notas) }\end{array}$ & $\begin{array}{l}\text { (2)DSF } 45 \\
\text { (notas) }\end{array}$ & $\begin{array}{l}{ }^{(3)} \text { DSF } 60 \\
\text { (notas) }\end{array}$ & $\begin{array}{l}\text { (4)Média Geral } \\
\text { (notas) }\end{array}$ \\
\hline DKB 230 PRO3 & 2,16 & $8,33 \mathrm{a}$ & $8,33 \mathrm{a}$ & $6,27 \mathrm{a}$ \\
\hline MG580 PW & 3,41 & $6,33 \mathrm{~b}$ & $6,66 \mathrm{c}$ & $5,47 \mathrm{a}$ \\
\hline DKB 290 PRO 3 & 2,75 & $6,0 \mathrm{c}$ & $6,0 \mathrm{c}$ & $4,91 \mathrm{a}$ \\
\hline 30F53 VYH & 0,91 & $5,83 \mathrm{c}$ & $6,33 \mathrm{c}$ & $4,36 \mathrm{a}$ \\
\hline MG600 PW & 0,66 & $5,83 \mathrm{c}$ & $6,16 \mathrm{c}$ & $4,22 \mathrm{a}$ \\
\hline DKB 177 PRO 3 & 0,5 & $5,33 \mathrm{c}$ & $6,66 \mathrm{c}$ & $4,16 \mathrm{a}$ \\
\hline RB 9110 PRO 2 & 0,33 & $5,66 \mathrm{c}$ & $6,5 \mathrm{c}$ & $4,16 \mathrm{a}$ \\
\hline AG 7000 PRO 2 & 1,91 & $4,16 \mathrm{~d}$ & $5,83 d$ & $3,97 \mathrm{a}$ \\
\hline DKB 390 PRO 2 & 2,5 & $4,33 d$ & $4,66 \mathrm{e}$ & $3,83 \mathrm{a}$ \\
\hline Formula VIP & 1,08 & $3,5 \mathrm{e}$ & $5,33 d$ & $3,30 \mathrm{~b}$ \\
\hline 30F35 VYH & 2 & $3,16 \mathrm{e}$ & $3,16 \mathrm{f}$ & $2,77 \mathrm{~b}$ \\
\hline P1680 YH & 1,5 & $1,83 \mathrm{f}$ & $1,83 \mathrm{~g}$ & $1,72 b$ \\
\hline AG 8088 PRO 2 & 0,75 & $1,66 \mathrm{f}$ & $1,66 \mathrm{~g}$ & $1,36 \mathrm{~b}$ \\
\hline Média Geral Bt & 1,57 & 4,76 & 5,31 & 3,88 \\
\hline AL BANDEIRANTE & 1,83 & $6,83 \mathrm{~b}$ & $7,33 \mathrm{~b}$ & $5,33 \mathrm{a}$ \\
\hline $\mathrm{F}_{2} \mathrm{DAS} 2 \mathrm{~B} 710 \times \mathrm{F}_{2} \mathrm{~A} 2555$ & 4,75 & $8,5 \mathrm{a}$ & $8,66 \mathrm{a}$ & $7,37 \mathrm{a}$ \\
\hline $\mathrm{F}_{2} \mathrm{DAS} 2 \mathrm{~B} 710 \times \mathrm{F}_{2} 30 \mathrm{~F} 35$ & 3,33 & $5,5 \mathrm{c}$ & $5,33 d$ & $4,88 \mathrm{a}$ \\
\hline $\mathrm{F}_{2} \mathrm{FORT} \times \mathrm{F}_{2} \mathrm{AG} 8060$ & 0,66 & $6,5 \mathrm{~b}$ & $6,33 \mathrm{c}$ & $4,58 \mathrm{a}$ \\
\hline$F_{2} 30 F 87 \times F_{2} A G 8060$ & 0,5 & $5,0 \mathrm{c}$ & $5,16 d$ & $3,77 b$ \\
\hline $\begin{array}{l}F_{2} D A S 2 B 710 x \\
F_{2} A G 8060\end{array}$ & 0,66 & $4,66 d$ & $4,5 \mathrm{e}$ & $3,99 \mathrm{~b}$ \\
\hline $\mathrm{F}_{2} \mathrm{~A} 2555 \times \mathrm{F}_{2} \mathrm{FORT}$ & 1,66 & 3,0 e & $3,0 f$ & $2,55 \mathrm{~b}$ \\
\hline $\begin{array}{l}\text { Média Geral } \\
\text { Convencional }\end{array}$ & 1,91 & 5,71 & 5,75 & 4,64 \\
\hline
\end{tabular}

Notas: Médias seguidas de mesma letra não diferem entre si pelo teste Scott- Knott ( $P$ $<0,05)$. Escala de notas de danos de lagarta-do-cartucho variando de 0 e 9.

Fonte: os autores. 
Tabela 6 - Valores e significância dos coeficientes de correlação fenotípica $\left(\mathrm{r}_{\mathrm{f}}\right)$. $2^{\circ}$ safra de 2018. Cáceres $-M T$.

\begin{tabular}{lccc}
\hline & DSF 30 & DSF 45 & DSF 60 \\
\hline DSF 30 & - & 0,383 & 0,306 \\
DSF 45 & & - & $0,95^{\star *}$ \\
DSF 60 & & & - \\
\hline
\end{tabular}

Notas: DSF: Danos de Spodoptera nas Folhas. ${ }^{* *}$ : significativo a 1\%, respectivamente, pelo teste $\mathrm{F}$.

Fonte: os autores.

\section{CONCLUSÕES}

Pode-se discriminar híbridos transgênicos resistentes à lagarta-docartucho, principalmente, da tecnologia Viptera ${ }^{\circledR}$. Enquanto, que o híbrido convencional $\mathrm{F}_{2} \mathrm{~A} 2555$ × $\mathrm{F}_{2} \mathrm{FORT}$ indicou na pré-avaliação a presença de mecanismo de resistência do tipo não-preferência. Para o controle de danos de lagartas infere-se o monitoramento de lagarta até o DSF45 para não causar perdas de produtividade de grãos.

\section{REFERÊNCIAS}

ANDOW, D. A. The risk of resistance evolution in insects to transgenic insecticidal crops. Collection of Biosafety Reviews, v. 4, p. 142-199, 2008.

ARAGÃO, F. J. L.; ANDRADE, P. P.; BORÉM, A. Variedades com eventos piramidados. Resistência de insetos-pragas a plantas geneticamente modificadas. Plantas geneticamente modificadas: Desafios e oportunidades para regiões tropicais. Ed. Aluízio Borém; Gustavo Almeida. Viçosa - MG. p. 139158, 2011.

ASSOCIAÇÃO DOS PRODUTORES DE SOJA E MILHO DO ESTADO DE MATO GROSSO. APROSOJA. A história do Milho. Cuiabá - MT: APROSOJA - MATO GROSSO, [s.d]. 1p. Disponível em: <http://www.aprosoja.com.br/sojae-milho/a-historia-do-milho>. Acesso em: 12 de setembro 2018.

BERNARDI, O. et al. Resistência de insetos-pragas a plantas geneticamente modificadas. Plantas geneticamente modificadas: Desafios e oportunidades para regiões tropicais. Ed. Aluízio Borém; Gustavo Almeida. Viçosa - MG. p. 179 $-204,2011$.

BOIÇA JUNIOR, A. L. et al. Atualidades em resistência de plantas a insetos. In: Tópicos em Entomologia Agrícola. Ed. Antonio Carlos Busoli...[et al.]. Jaboticabal: Maria de Lourdes Brandel, p. 207- 224, 2013.

CÂMARA, T. M. M. et al. Parâmetros genéticos de caracteres relacionados à tolerância à deficiência hídrica em milho tropical. Bragantia, v. 66, n. 4, p. 595603, 2007. 
CONAB - COMPANHIA NACIONAL DE ABASTECIMENTO. Acompanhamento da safra brasileira de grãos 2018/19. Décimo segundo levantamento, 2019. Disponível em: <https://www.conab.gov.br/infoagro/safras> Acesso em: 12 set. 2019.

CRUBELATI-MULATI, N. C. S. et al. Evaluation of resistance to Spodoptera frugiperda in sweet and field corn genotypes. Maydica, v. 64, n. 3, 2019.

CRUZ, I. A lagarta do cartucho na cultura do milho. Sete Lagoas: EMBRAPA, Centro Nacional de Pesquisa de Milho e Sorgo, 1995. 45p.

DAVIS, F. M.; NG, S. S.; WILLIAMS, W. P. Visual rating scales for screening whorlstagecorn for resistance to fall armyworm. Mississippi: Agricultural and Forest Experiment Station, n. 186, 1992. 9p. Technical Bulletin.

FERNANDES, O. D. et al. Efeito do milho geneticamente modificado MON810 sobre a lagarta-do-cartucho Spodoptera frugiperda (J. E. Smith, 1797) (Lepidoptera: Noctuidae). Revista Brasileira de Milho e Sorgo, v. 2, n. 2, p. 2535, 2003.

HANASHIRO, R. K. Dialelo de populações F2 de milho (Zea mays L.) visando produtividade, resistência a Spodoptera frugiperda e a Helicoverpa zea. Campinas - SP: Instituto Agronômico de Campinas - IAC, 2014. 113p.

MENDES, S. M.; WAQUIL, J. M. Uso do milho Bt no manejo integrado de lepidópteros-praga: recomendações de uso Sete Lagoas: Embrapa Milho e Sorgo, 2009. 8 p. (Embrapa Milho e Sorgo. Comunicado técnico, 170).

MORAES, A. R. A. M; LORENÇÃO, A. L; PATERNIANI, M. E. A.G. Z. Resistência de híbridos de milho convencionais e isogênicos transgênicos a Spodoptera frugiperda (Lepidoptera: Noctuidae). Bragantia, v. 74, n. 1, p. 50-57, 2015.

OLIVEIRA, T. A. Resistência de Híbridos Transgênicos e Convencionais de Milho à Lagarta-do-Cartucho. Cáceres-MT. Universidade do Estado de Mato Grosso - UNEMAT, 2018. 20p.

OTA, E. C. et al. Desempenho de cultivares de milho em relação à lagarta-docartucho. Bragantia, v. 70, n. 4, p.850-859, 2011.

PEREIRA-FILHO, I. A.; BORGHI, E. P. Sementes de milho no Brasil - A Dominância dos Transgênicos. 21. ed. Sete Lagoas - MG: Embrapa Milho e Sorgo, 2018. 31p. (Documentos, 223).

SCAPIM, C. A.; CARVALHO, C. G. P. de; CRUZ, C. D. Uma proposta de classificação dos coeficientes de variação para a cultura do milho. Pesquisa Agropecuária Brasileira, v. 30, n. 5, p. 683-686, 1995. 
SOBERÓN, M.; GILL, S. S.; BRAVO, A. Signaling versus punching hole: How do Bacillus thuringiensis toxins kill insect midgut cells? Cellular and Molecular Life Sciences, v. 66, n. 8, p. 1337-1349, 2009. 